\title{
Spatial and temporal scales of coronal magnetic restructuring in CME initiation
}

\author{
Yayuan Wen ${ }^{1}$, Jingxiu Wang ${ }^{1}$, Dalmiro Joreg Filipe Maia ${ }^{1,2}$, Yuzong \\ Zhang $^{1}$, and Hui Zhao ${ }^{1}$ \\ ${ }^{1}$ National Astronomical Observatories, Chinese Academy of Sciences, Beijing 100012, China \\ email: yayuanwen@ourstar.bao.ac.cn \\ ${ }^{2}$ CICGE, Observatório Astronómico Professor Manuel de Barros, Faculdade de Ciências da \\ Universidade do Porto, 4430-146 Vila Nova de Gaia, Portugal
}

\begin{abstract}
Based on the concept that the CMEs are the results of coronal magnetic restructuring or reconfiguring, we analyze the December 18, 2000 event by using Nançay Radioheliograph $(\mathrm{NRH})$ images and combining the multi-spatial scale observations. We investigated the onset, duration and position of the radio emissions in relation to EUV dimming and the inferred CME onset, with the purpose to estimate the spatial and temporal scales of the coronal restructuring, thus, to understand the physics of the initiation and development of the CME.
\end{abstract}

Keywords. Sun:coronal mass ejections (CMEs), Sun:radio radiation

\section{Observation}

The December 18, 2000 event is associated with a GOES C7.0 class flare occurring at 11:03 UT inside NOAA Active Region (AR) 9269. The CME associated with this flare was first seen in C2 at 11:50 UT. It has been identified that the early on-disk development of this CME is characterized by the appearance of series distinct radio bursts of Type II and Type IV at $164 \mathrm{MHz}$ by NRH (Kerdraon et al. 1997), in addition to the EUV dimming (Thompson et al. 1998) seen in EIT images. All the identified radio sources are denoted in Figure 1. We find that the successive appearance of the episodic bursts guided the expansion and propagation of EUV dimming. It is likely that each radio burst outlines the EUV wave fronts. It is remarkable that allowing the projection effects all the burst sources are in the dimming regions. To understand the association of the radio bursts with the magnetic flux distribution and connectivity, all the identified radio sources are marked in the MDI magnetogram and magnetic field extrapolation at 09:36 UT in Figure 2. AR 9269 was near the Sun's center on the disk. An unipolar region is located on the north of AR 9269. Majority of the radio bursts appeared in the conjunction of many sets of magnetic loop systems, a common separatrix in the northern hemisphere. Their locations were closely related to discrete sets of magnetic loop systems.

\section{Results}

The analysis of the event demonstrates that the early on-disk initiation of CMEs is manifested by episodic non-thermal radio bursts. The radio bursts appeared in the EUV dimming or on-going dimming regions whose position and extent coincided with the CME span in LASCO C2 field of view. These bursts have an average duration of 70 seconds. They are separated one another by approximate 2 minutes. The overall time scale of all the radio sources is about 15 minutes. The individual radio emission seems to shift and expand at the speed of the fast magneto-acoustic waves in the local corona; 


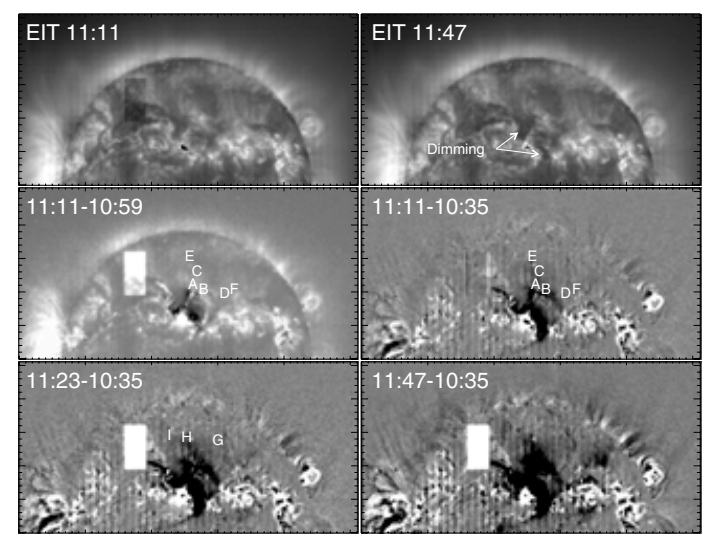

Figure 1. The EIT and the EIT running or base difference images showing the larger dimming region. The positions of radio bursts at $164 \mathrm{MHz}$ are marked by the letters.
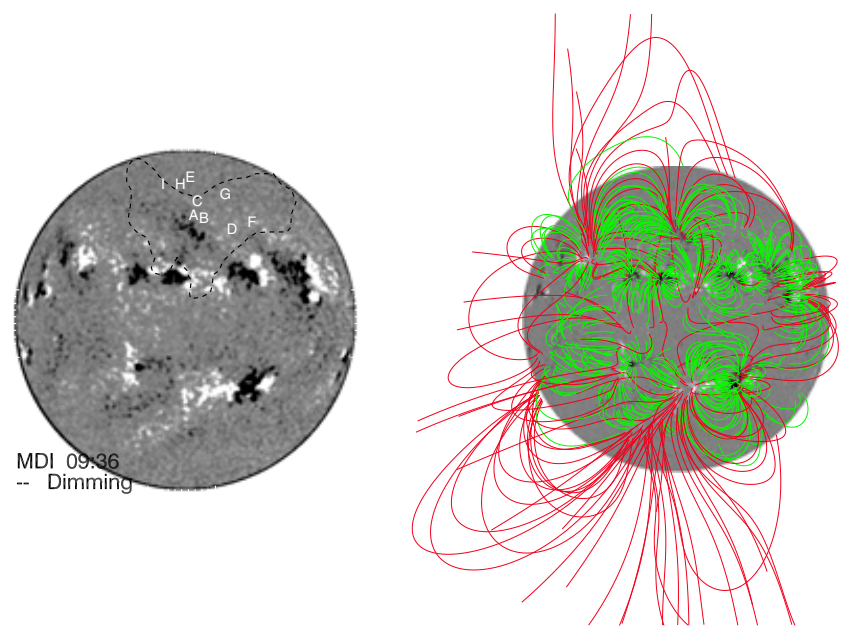

Figure 2. (Left) The positions of radio bursts of the NRH at $164 \mathrm{MHz}$ are marked in the SOHO MDI magnetogarm. (Right) The extrapolated magnetic filed lines showing the pre-CME magnetic topology of the whole Sun.

while the non-thermal radio emission as a whole appear episodically to correspond to the successive restructuring. If we define the CME triggering speed by dividing the overall spatial scale by the time scale of all the radio bursts, then the triggering speed falls in the range of $300-400 \mathrm{~km} / \mathrm{s}$. This implies that the general process of coronal restructuring and reconfiguring takes place at a speed slower than either the Alfvenic or acoustic speed in the corona. The triggering speed represents how fast the magnetic topology changes from close to open field configuration in the CME source regions. In this sense, we might regard the triggering speed as a type of speed of 'topology waves'.

This work is supported by National Natural Science Foundation of China (10233050) and National Key Basic Research Science Foundation of China (TG2000078404).

\section{References}

Thompson, B. J. et al. 1998, Geophys. Res. Lett. 25, 2465

Kerdraon, A., \& Delouis, J.-M. 1997, in Coronal Physics from Radio and Space Observations, ed. G. Trottet (Springer: Berlin), 483, 192 